Review

\title{
Exosomes in osteoarthritis and cartilage injury: advanced development and potential therapeutic strategies
}

\author{
Quanfa Zhou1, Youzhi Cai ${ }^{1,2}{ }^{\bowtie}$, Yangzi Jiang 3 and Xiangjin Lin ${ }^{1}$ \\ 1. Department of Orthopaedic and Center for Sports Medicine, the First Affiliated Hospital, School of Medicine, Zhejiang University, Zhejiang, Hangzhou, \\ China \\ 2. Dr. Li Dak Sum \& Yip Yio Chin Center for Stem Cell and Regenerative Medicine, School of Medicine, Zhejiang University, Hangzhou, China. \\ 3. Institute for Tissue Engineering and Regenerative Medicine, School of Biomedical Sciences, Faculty of Medicine, The Chinese University of Hong Kong, \\ Hong Kong S.A.R., China \\ $\square$ Corresponding author: Youzhi Cai, M.D., Orthopaedic Department and Sports Medical Center, First Affiliated Hospital of Zhejiang University, Building for \\ 79 Qingchun Road, Shangcheng District, Hangzhou, China, 31003. Email: caiyouzhi@zju.edu.cn.
}

(๑) The author(s). This is an open access article distributed under the terms of the Creative Commons Attribution License (https://creativecommons.org/licenses/by/4.0/). See http://ivyspring.com/terms for full terms and conditions.

Received: 2019.11.03; Accepted: 2020.03.10; Published: 2020.03.31

\begin{abstract}
Articular cartilage injury is a common clinical problem, which can lead to joint dysfunction, significant pain, and secondary osteoarthritis (OA) in which major surgical procedures are mandatory for treatment. Exosomes, as endosome-derived membrane-bound vesicles, participating in intercellular communications in both physiological and pathophysiological conditions, have been attached great importance in many fields. Recently, the significance of exosomes in the development of OA has been gradually concerned, while the therapeutic value of exosomes in cartilage repair and OA treatment has also been gradually revealed. The functional difference of different types and derivations of exosomes are determined by their specific contents. Herein, we provide comprehensive understanding on exosome and $\mathrm{OA}$, including how exosomes participating in $\mathrm{OA}$, the therapeutic value of exosomes for cartilage injury/OA, and related bioengineering strategies for future therapeutic design.
\end{abstract}

Key words: exosome, osteoarthritis, cartilage, exosomal RNA, stem cells, cellular and exosomal therapies

\section{Introduction}

The occurrence of osteoarthritis (OA) implies an imbalance in degradation-synthesis of chondrocytes, extracellular matrix (ECM) and subchondral bone, but the detailed molecular mechanisms remain unclear. Exosomes, as a type of extracellular vesicles (EVs), play a role in tissue-tissue and cell-cell communications in homeostasis and diseases, and the mechanism of exosomal involvement in the development of OA has only recently been reported. Thus, understanding how exosomes participate in the process of OA will help to seek a novel way for developing the treatment of OA.

Traditional non-surgical treatments for OA can improve symptoms but cannot restore articular cartilage regeneration or modify degenerative processes ${ }^{[1]}$. Although surgical arthroplasty results in long-term functional improvement and improves quality of life, this only suit for the end stage of the diseases, and instability and infection are the most common limitations, necessitating further revision surgery. Cell-based therapy, especially mesenchymal stem cells (MSCs), has facilitated rapid advances in regenerative medicine for $\mathrm{OA} /$ cartilage injury in recent years. However, the clinical applications of stem cells have raised considerable concerns, such as teratomas, immune rejection, non-stable between batches and dose-dependent effect [2-3]. The bio-effects of MSCs mainly through paracrine, especially via the exosomes they produced [4]. Thus, exosome-based therapy may be a promising substitute for stem cell therapy on cartilage injury/OA, which provides the possibility of "cell-free" therapy. 
This review will focus on the biological characteristics of exosomes, and the involvement of exosomes in pathology of OA will be discussed in detail. We will also discuss the evidence that shows how exosomes can be used as "cell-free" therapy for $\mathrm{OA} /$ cartilage injury, and the detailed exosome-based tissue engineering strategies. Finally, we will also discuss future development in this exciting field.

\section{The biological characteristics of exosomes}

Exosomes are endosome-derived membranebound vesicles with diameters of 50-150 nm, released by cells in all living systems in physiological and pathophysiological conditions [5]. Exosomes originate from the endosomes that are generated by endocytosis of the cytoplasmic membrane. Later, inside the cell, cargoes such as mRNA or proteins accumulate inside the endosomes, thus forming multivesicular endosomes or intraluminal vesicles [6]. After further processing, exosomes are finally released through membrane fusion [7]. At early ages, exosomes were regarded as useless cellular metabolic waste, but it has since been recognized that exosomes carry proteins, lipids, and nucleic acids, including mRNA, microRNA (miRNA), and long non-coding RNA, which play important roles in intercellular communications and cellular immune response ${ }^{[8]}$.

The biogenesis and release of exosomes is a complex symphony involving a series of factors, representatively including the endosomal sorting complexes required for transport (ESCRT), ALIX (also known as programed cell death 6-interacting protein, PDCD6IP), phospholipase, vacuolar protein sorting-associated 4 (VPS4), Rab GTPase proteins, sphingomyelinase and ceramide. Recently, the inhibition effect of exosome release was reported by sustained activation of mechanistic target of rapamycin complex 1 (mTORC1) in both cells and animal models, while inhibition of mTORC1 stimulated the release of exosomes, which occurred concomitantly with autophagy ${ }^{[9]}$.

The recognition of membrane receptors is the basis for the exosome-cell reaction and interactions. The activation of the receptors and subsequently lead to the activation of associated signaling pathways, subsequently the fusion of exosomes with plasma membrane or the endocytosis of exosomes occurs. Through above-mentioned internalization pathways, exosomes can either release their cargos into the recipient cells to exert their functions, or be directly degraded by lysosome for recycling [10]. Thus, the function and biological characteristic of exosomes are determined by their specific contents.

\section{Pathological related exosomes in OA}

Although many beneficial biological effects are based on exosomes, in OA joint, chondrocytes, synoviocytes and immune cells may also deliver pathogenic signals to each other through exosomes, and these communications break the balance of joint microenvironment and further aggravate OA. In OA tissues, an increased number of immune cells associated with pro-inflammatory cytokine expression, including tumor necrosis factor (TNF)- $\alpha$, interleukin (IL)-1 $\beta$, IL-6 and IL-22, was detected from OA synovial tissue [11], while matrix metalloproteinase (MMP), is thought to be the major mediator of ECM breakdown, which causes the majority of the pathologies seen in OA [12]. Analysis the relationship between exosomes and these important pathological factors may provide insights for our understanding of OA pathology (Figure 1).

\section{Exosomes in OA pathology}

Exosomes in OA joint fluid were firstly analyzed by many research groups and it indeed usually links with pathological effects. Exosomes from OA joint fluid could influence the gene expression of chondrocytes negatively. Kolhe et al treated healthy articular chondrocytes with OA-derived exosomes, showing decreased anabolic genes expression and elevated expression of catabolic and inflammatory genes [13]. In addition, exosomes from OA joint fluid could activate inflammatory cells. Domenis et al found that synovial fluid-derived exosomes significantly stimulated the release of several inflammatory cytokines, chemokines and metalloproteinases by M1 macrophages [14]. Incubating macrophages with exosomes from synovial fluid of $\mathrm{OA}$, Song et al demonstrated that the exosomes could induced proliferation and osteoclast formation without macrophage colony-stimulating factor (M-CSF) and receptor activator of nuclear factor kappa-B ligand (RANKL) [15].

Furthermore, some researches have studied the role of exosomes in the communication between chondrocytes and other cells in OA. Nakasa et al reported that when exosomes derived from chondrocytes treated with IL-1 $\beta$ were applied to fibroblast-like synoviocytes, there was a nearly three-fold increase in MMP-13 production as compared with exosomes derived from chondrocytes without IL-1 $\beta$ stimulation [16]. In turn, Kato et al found exosomes from IL-1 $\beta$ stimulated human synovial fibroblasts (SFB) up-regulated MMP-13 and ADAMTS-5 expression in articular chondrocytes and down-regulated collagen alpha 1 (Col2a1) and aggregated proteoglycan core protein (ACAN) in vivo [17]. In one chip-assay study, 50 miRNAs were 
identified in exosomes in response to IL-1 $\beta$ stimulated SFB compared to in non-stimulated SFB, and among them, miR-4454 and miR-199b are related to inflammatory stimulation ${ }^{[18]}$ and cartilage formation [19], respectively. $\mathrm{Ni}$ et al found the exosome-like vesicles from IL-1 $\beta$-pretreated chondrocytes could promote mature IL-1 $\beta$ production of macrophages [20].

Kolhe et al reported the differential expression of miRNAs between exosomes from OA synovial fluid and normal synovial fluid [13]. They found the gender differences of the expression of exosomal miRNAs, with only one (miRNA-504) existing in both genders. Interestingly, the authors also demonstrated that female OA-specific exosomal-miRNAs from synovial fluid were estrogen-responsive and targeted toll-like receptor (TLR) signaling pathways, which might relate to the increased prevalence of $\mathrm{OA}$ in post-menopausal females. Furthermore, the patients with OA had lower levels of exosomal miR-193b in plasma than normal control subjects [21].

The differential expression of miRNA of exosomes from chondrocytes in the inflammatory microenvironment which related to $\mathrm{OA}$ has been reported recently. Exosomal miR-92a-3p expression was significantly reduced in the OA chondrocyte- secreted exosomes. Mao et al found that miR-92a-3p suppressed the activity of a reporter construct containing the 3'-UTR and directly targeted WNT5A in both MSCs and chondrocytes [22], and WNT5A plays important role in both chondrogenic differentiation and cartilage degradation [23]. Mao et al also found another exosomal miRNA (miR-95-5p) was down-regulated in OA chondrocytes [24]. Furthermore, they demonstrated that miR-95-5p could regulate cartilage development and homoeostasis by directly targeting histone deacetylase (HDAC)2/8. HDAC2/8 tends to impede cartilage development by inhibiting the expression of cartilage-specific genes [25].

Many exosomal miRNAs are founded related to OA development, there are exosomal proteins related to OA too. Recently, Varela-Eirín et al found that overexpression of channel protein connexin 43 (Cx43) in chondrocytes increased senescence and exosomal Cx43 levels [26]. In this study, OA chondrocytes showed increased levels of Cx43 within their EVs in comparison to the EVs isolated from healthy donors.

\section{The Diagnostic Value of Exosomes for OA}

Theoretically, the variation levels of exosomal miRNAs or proteins mentioned above have potential

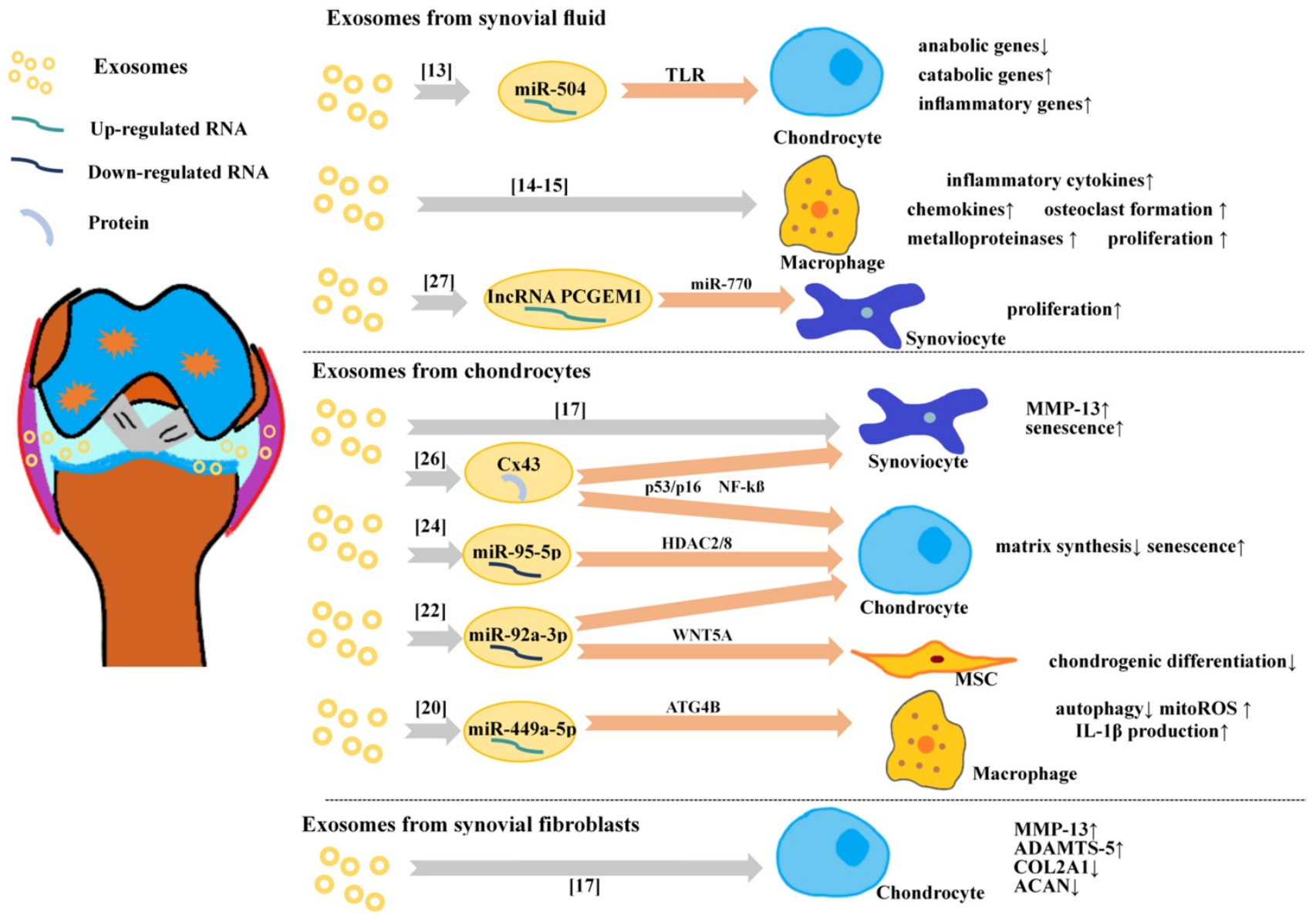

Figure 1. Exosomes involved in pathology of $O A$. 
to become biomarkers for OA diagnose. However, the value of exosomes as a diagnostic tool of $\mathrm{OA}$ is still under discussion. Recently, Zhao et al investigated the diagnostic value of exosomes from plasma and from synovial fluid in patients with OA in distinguishing the early stage of OA from progressive stage of OA [27]. They found that in synovial fluid, the expression of exosomal lncRNA PCGEM1 was markedly higher in late-stage $\mathrm{OA}$ than in early-stage $\mathrm{OA}$, and markedly higher in early-stage OA than normal controls, demonstrating that exosomal lncRNA PCGEM1 from synovial fluid might be a powerful indicator in distinguishing early-stage from late-stage OA. IncRNA PCGEM1 acts as a sponge lncRNA targeting miR-770 and then stimulates proliferation of osteoarthritic synoviocytes [28]. However, the general evaluation of plasma and synovial fluid exosome was not viable for identifying OA stages, and the clinical application of exosomes in the diagnosis of $\mathrm{OA}$ remains challenging.

\section{Exosomes with therapeutic effects for OA and cartilage injury}

\section{The exosomes from mesenchymal stem cells}

Exosomes are one of the key secretory products of MSCs, resembling the effect of parental MSCs, and can be directly used as therapeutic agents for various disease models, such as cutaneous wound[29], osteonecrosis of the femoral head [30], and neurological injury[31]. Recent years, exosomes from different unmodified MSCs were reported to have exact therapeutic effects on OA and cartilage injury (Figure 2).

\section{Exosomes from different types of MSCs}

Cartilage regeneration from bone marrow mesenchymal stem cells (BMSCs) is the core of microfracture technology. Exosomes from BMSCs have been studied in recent years. Cosenza et al demonstrated the protective effect of exosomes from BMSCs in the collagenase-induced OA model [32]. Furthermore, they found exosomes from BMSCs could restore the expression of chondrocyte markers (type II collagen, aggrecan) while inhibiting catabolic (MMP-13, ADAMTS5) and inflammatory (iNOS) markers in OA-like chondrocytes in vitro. Zhu et al also revealed that BMSCs derived exosomes could protect chondrocytes from apoptosis and senescence [33]. Furthermore, Qi et al observed the uptake of exosomes from BMSCs by chondrocytes [34], and exosomes from BMSCs could inhibit mitochondrialinduced apoptosis of chondrocytes in response to IL-1 $\beta$, with p38, ERK, and Akt pathways involved.

\section{In vivo}

Embryonic MSC-derived exosomes

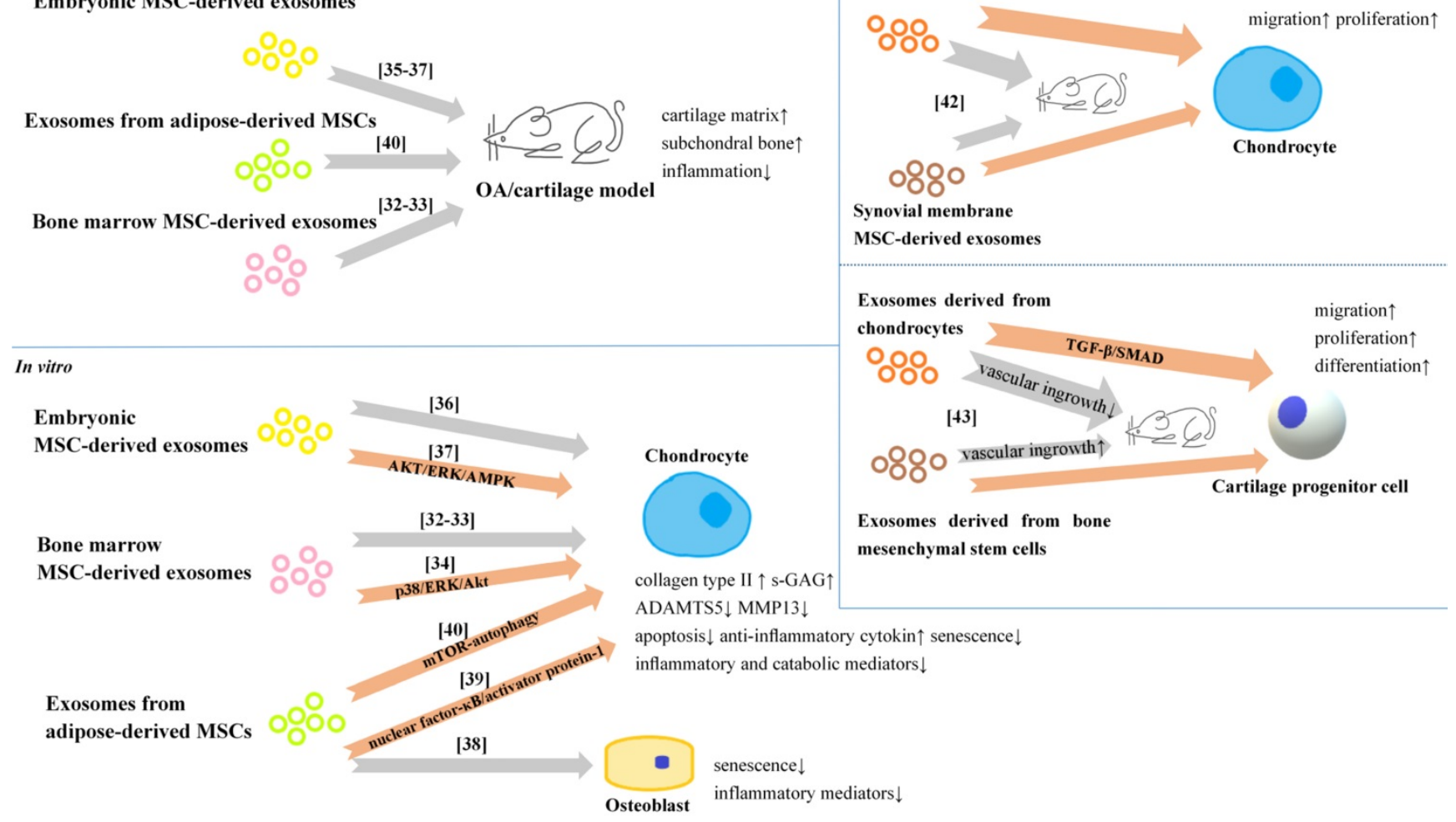

Figure 2. Exosomes from unmodified cells for OA therapy/cartilage. 
Exosomes from embryonic MSC (ESCs) have shown the potential of alleviating matrix degradation and promoting cartilage repair in some animal models [35-37]. Further in vitro, exosomes from ESCs could maintain the chondrocyte phenotype by increasing collagen type II synthesis and decreasing ADAMTS5 expression [36]. Zhang et al demonstrated that the joint repair effects of exosomes from ESCs could be attributed to adenosine activation of protein kinase B(AKT), extracellular signal-regulated kinase (ERK) and adenosine monophosphate-activated protein kinase (AMPK) signaling [37]. In addtion, they also found that the joint repair effects might be related to exosomal CD73 expression which can convert extracellular AMP to adenosine, as well as exosomal transforming growth factor- $\beta$ (TGF- $\beta$ ) and insulin growth factor (IGF).

Due to the feasibility to obtain human infrapatellar fat pad from OA patients by arthroscopy, using exosomes from adipose-derived mesenchymal stem cells (ADSCs) has gradually gain more attentions. Tofiño-Vian et al found exosomes from ADSCs could down-regulated senescence-associated $\beta$-galactosidase activity and the accumulation of $\gamma \mathrm{H} 2 \mathrm{AX}$ foci, and reduced the production of inflammatory mediators from OA osteoblasts [38]. They also reported that exosomes from ADSCs could reduce the production of inflammatory and catabolic mediators from OA chondrocytes stimulated with IL-1 $\beta$ [39]. The chondroprotection role could be the consequence of a lower activation of nuclear factor- $\mathrm{kB}$ and activator protein-1. In other study, $\mathrm{Wu}$ et al attributed the cartilage protection of exosomes from ASCs to the high level of exosomal miR-100-5p [40], because the exosomal miR-100-5p could bind to the 3 '-untranslated region (3'UTR) of mTOR, then significantly enhance autophagy level in OA chondrocytes via mTOR inhibition.

\section{Therapeutic contents in exosomes}

It is significant to figure out the therapeutic contents in exosomes. Recently, Liu et al verified that lncRNA KLF3-AS1 in human MSCs and exosomes derived from human MSCs (MSC-Exos) by qRT-PCR analysis [41], and it might be the key molecule with therapeutic effect. In their study, treating rat chondrocytes with the MSC-Exos whose lncRNA KLF3-AS1 expression was knocked down could reverse the normal chondroprotection of MSC-Exos. The knee joint cartilage damage of rat OA model was also deteriorated by the MSC-Exos without lncRNA KLF3-AS1 expression. These data suggested that the therapeutic effect of MSC-Exos on OA is related to the newly discovered exosomal lncRNA KLF3-AS1.

\section{Comparison of the effects of exosomes}

More importantly, exosomes from different cell types may have different effects, and this topic is still under investigation. Zhu et al compared the effects of exosomes secreted by synovial membrane MSCs (SMMSC-Exos) and exosomes secreted by induced pluripotent stem cell-derived MSCs (iMSC-Exos) in treating OA [42]. They found both exosomes attenuated OA in the mouse OA model, but iMSC-Exos had a superior therapeutic effect compares to SMMMSC derived exosomes, and iMSC-Exos exerted a stronger effect on chondrocyte migration and proliferation in vitro. In another study, Chen et al found that exosomes derived from chondrocytes (CC-Exos) increased collagen deposition and minimized vascular ingrowth in engineered constructs, and efficiently and reproducibly developed into cartilage, while the BMSC-Exos treated tissue engineered construct was characterized with hypertrophic differentiation accompanied by vascular ingrowth [43]. In vitro, CC-Exos could stimulate cartilage progenitor cells proliferation and significantly promoted chondrogenesis-related factors at the mRNA and protein levels.

\section{Exosomes from molecular engineered cells}

The strategies of utilizing exosome loading technology to obtain customized drug-loaded exosomes are gradually applied in the field of OA therapy (Figure 3). There are two ways to load drugs into exosomes, one is to load drugs into the donor cell of exosomes, such as using transfection and co-incubation; the other is to load drugs into exosomes after they are secreted, such as direct mixing, which the loading efficacy is a big concern. At present, most researchers prefer to obtain the exosomes for OA therapy with high expression of miRNA or lncRNA from modified MSCs [22,44-46]. Exosomal miR-92a-3p [22], exosomal lncRNA-KLF3AS1 [44], exosomal miR-140-5p [45] and exosomal miR-320c [46] from transfected MSCs have been reported to have significant therapeutic effects on OA in vivo and in vitro. Furthermore, it was reported that primary chondrocytes also could be modified to serve as the donor cells of exosomes [24]. In that study, exosomes derived from miR-95-5p-overexpressing primary chondrocytes promoted cartilage developpment and cartilage matrix expression by directly targeting $\mathrm{HDAC} 2 / 8$ in MSCs induced to undergo chondrogenesis and chondrocytes, respectively. In addition to transfection, treating the donor cells with proper growth factors such as TGF $\beta 1$ can also improve the productivity of therapeutic exosomes ${ }^{[4]}$. 


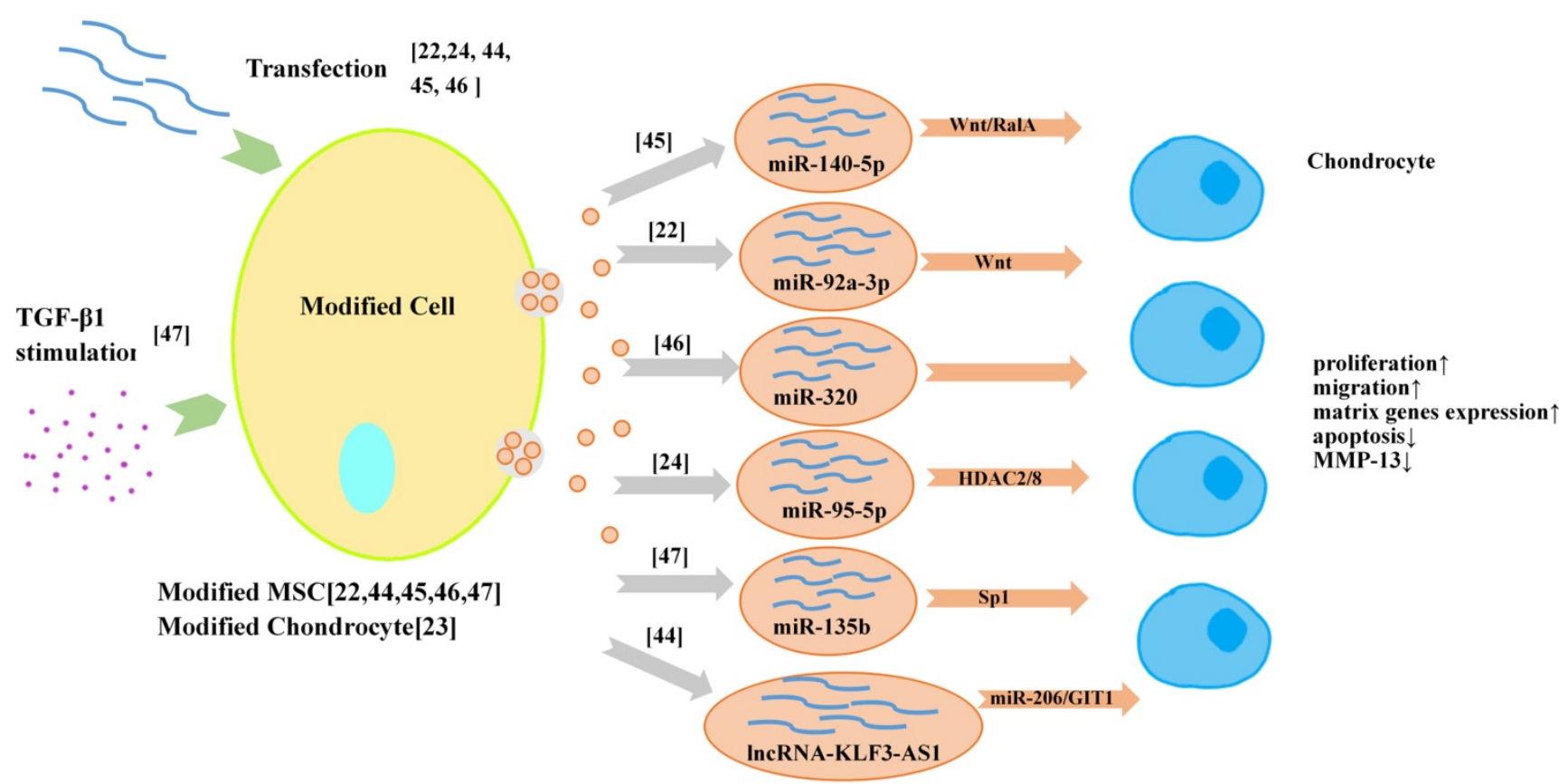

Figure 3. Exosomes from modified cells for OA therapy/cartilage repair.

\section{Tissue engineering strategies for exosomes}

Exosome-based tissue engineering technology representing an advanced strategy, has attracted increasing attention in many fields (Table 1). In this section, we will focus on some representative exosome-based tissue engineering strategies (Figure 4) and discuss their application potential in OA therapy/cartilage repair.

\section{D culture for exosome generation}

In a multicellular organism, tissue cells are highly organized in a 3-dimensional (3D) fashion and

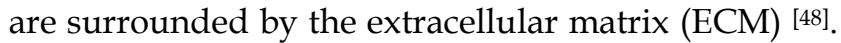
Under 2-dimensional (2D) conditions, cells lack the in vivo spatial polarization and architecture, leading to changes in cellular morphology, proliferation and functionalities, such as the processing and function of EVs [49]. Therefore, for better understanding the roles of exosomes in OA development and treatment, the technology of 3D culture, has attracted increasing attention in order to improve on the inadequate reproduction of the in vivo microenvironment by $2 \mathrm{D}$ culture.

Some studies have reported that exosome produced by 3D cultures (Exos/EVs-3D) has more advantages than exosome produced by $2 \mathrm{D}$ cultures (Exos/EVs-2D) [50-57] in terms of the size, content, function and production efficiency of exosomes. The functions of exosomes differ according to their content, while Exos/EVs-3D contain a different profile of proteins and genetic materials compared to Exos/EVs-2D [52]. The enhanced production efficiency of Exos/EVs-3D might relate to the reduction of expression of F-actin in 3D culture [53]. However, there is also one study reporting no difference in functions regarding the $2 \mathrm{D} / 3 \mathrm{D}$ derivation of exosomes ${ }^{[54]}$.

Table 1. Tissue Engineering Strategies for Osteoarthritis and Cartilage Injury

\begin{tabular}{|c|c|c|}
\hline $\begin{array}{l}\text { Tissue-Engineering } \\
\text { Technology }\end{array}$ & Advantages for exosomes & Related reports \\
\hline 3D culture & $\begin{array}{l}\text { Regulating the biological } \\
\text { characteristics; }\end{array}$ & $\begin{array}{l}\text { Size[50-51]; } \\
\text { Content[50-52, 55-57]; } \\
\text { Function[50, 52, 54-55]; } \\
\text { Production efficiency[50, } \\
\text { 52-53, 55-57] }\end{array}$ \\
\hline \multirow[t]{3}{*}{ Nanoparticles } & $\begin{array}{l}\text { Changing biological } \\
\text { characteristics }\end{array}$ & $\begin{array}{l}\text { Regulating exosome release } \\
\text { of parent cells }[58,59] ; \\
\text { Regulating content of } \\
\text { exosome[60]; }\end{array}$ \\
\hline & Surface modification; & $\begin{array}{l}\text { Improving the targeting } \\
\text { ability of exosomes[61]; }\end{array}$ \\
\hline & $\begin{array}{l}\text { Combining with } \\
\text { exosomes for improving } \\
\text { deficiencies; }\end{array}$ & $\begin{array}{l}\text { Efficiently encapsulating } \\
\text { large plasmids[62]; }\end{array}$ \\
\hline \multirow[t]{2}{*}{ 3D biomaterials } & $\begin{array}{l}\text { Exosome retention and } \\
\text { sustained release function } \\
\text { as working platforms; }\end{array}$ & $\begin{array}{l}\text { Bone regeneration[63]; } \\
\text { Cardiac repair[65]; } \\
\text { Vascular disease[66]; } \\
\text { Wound healing[67]; } \\
\text { Cartilage repair[71]; }\end{array}$ \\
\hline & $\begin{array}{l}\text { Increasing the stability of } \\
\text { content; }\end{array}$ & $\begin{array}{l}\text { Increasing the stability of } \\
\text { proteins and miRNAs in } \\
\text { exosomes[68]; }\end{array}$ \\
\hline \multirow[t]{2}{*}{ 3D printing } & $\begin{array}{l}\text { Designing more } \\
\text { optimized 3D culture } \\
\text { microenvironment; }\end{array}$ & $\begin{array}{l}\text { Improving the productivity } \\
\text { and functions of } \\
\text { exosomes[57]; }\end{array}$ \\
\hline & $\begin{array}{l}\text { Designing scaffolds with } \\
\text { more optimized } \\
\text { geometric structure; }\end{array}$ & $\begin{array}{l}\text { Bone defect repair[72]; } \\
\text { Osteoangiogenesis[73]; } \\
\text { Cartilage repair[74]; }\end{array}$ \\
\hline
\end{tabular}




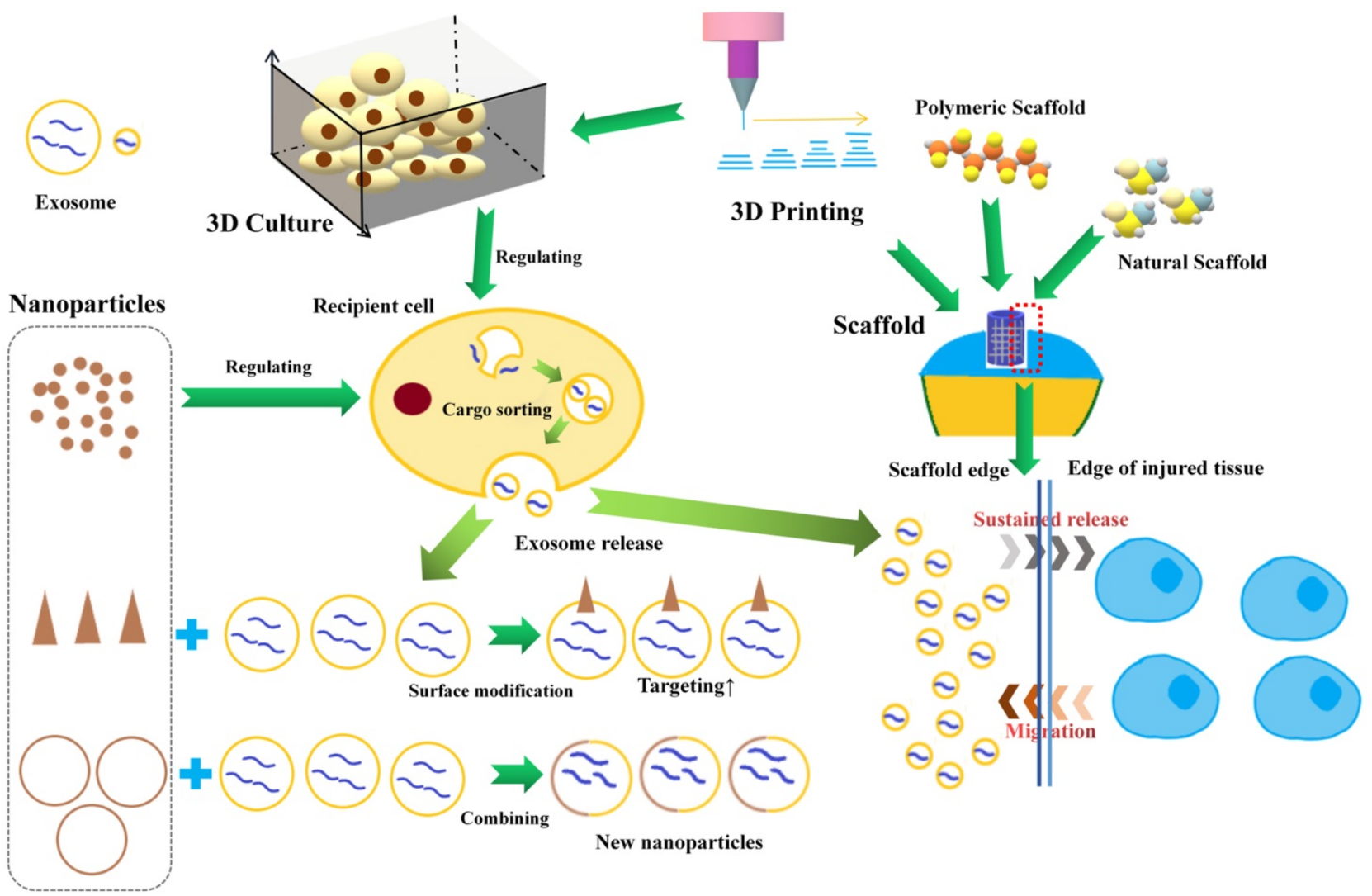

Figure 4. Exosome-based engineering strategy.

\section{Nanoparticles}

Nanoparticles can influence and assist the production and function of exosomes. Kasper et al found silica nanoparticles could decrease secretion of ICAM/E-selectin bearing exosomes/microvesicles when exposed to the inflamed endothelium [58]. Roma-Rodrigues et al found that gold nanoparticles (AuNPs) functionalized with thiolated oligonucleotides anti-RAB27A could decrease the release of exosomes due to specific gene silencing [59]. In addition, nanoparticles can also affect the sorting of exosomal cargos. Liang et al demonstrated that Sphk2 gene silencing induced by siRNA loaded nanoparticles could reduce miRNA-21 sorting into exosomes [60]. Nanoparticles can also enhance the targeting ability of exosomes. Khongkow et al reported that the surface modification of AuNPs with brain-targeted exosomes derived from genetically engineered mammalian cells enhanced their transport across the blood-brain-barrier [61]. Moreover, nanoparticles can also enhance the drug loading ability of exosomes. In order to solve the problem of low efficiency of exosomes in encapsulation of large nucleic acids, Lin et al developed a kind of hybrid nanoparticle combining exosome and liposome via simple incubation, which efficiently encapsulates large plasmids ${ }^{[62]}$.

\section{D biomaterials and exosome retention}

3D scaffolds could be further used as working platforms for the exosomes while attempting to control the release of exosomes in the tissue repaired area [63-64]. Hydrogel has been widely used as 3D scaffold due to their unique features, such as high water content, biocompatibility, swelling behavior, and modulated 3D networks, in many restorative areas, such as cardiac repair [65], vascular disease [66], wound healing [67]. Recently, Zhang et al reported that chitosan hydrogel could notably increase the stability of proteins and miRNAs in exosomes [68]. In OA therapy, hydrogel materials also have been widely to better fill the cartilage defect and provide a mode and mechanical support for cartilage regeneration [69]. Interestingly, hydrogel materials have been proven to have good exosome retention and sustained release function. Schneider et al found that many types of proteins secreted by chondrocytes encapsulated within photoclickable poly(ethylene glycol) hydrogels have been reported to be present within cell-secreted exosomes [70]. They suggested that the ability of diffusing through the hydrogel of smaller exosomes contributed to the results. Liu et al exploited a photoinduced imine crosslinking hydrogel glue as an exosome scaffold to prepare an acellular tissue patch for cartilage regeneration [71]. They found that most of 
the encapsulated exosomes were retained inside the hydrogel $(>90 \%)$ after immersing in PBS for 14 days. In addition, the tissue patch could release low concentration of exosomes showing positive regulation to the surrounding cells.

\section{D bio-printing}

3D printing has been well used in cartilage tissue repair, and to adopt the great bioeffects of exosome into 3D printing technology, there are two break points for further development. One is to improve the productivity and functions of exosomes by using optimized 3D culture microenvironment, which can be precisely designed and printed with 3D bio-printers [57]. The other is to design advanced bio-mimic scaffolds with more optimized geometric structure and better incorporate with exosomes, thus enhancing the therapeutic effects of exosomes or EVs [72-73]. For instance, Chen et al reported the interaction between 3D printing scaffold and exosome in cartilage repair ${ }^{[74]}$. They fabricated a 3D printed cartilage ECM/gelatin methacrylate/exosome scaffold with radially oriented channels using desktop-stereolithography technology. They found the 3D printed scaffold could effectively retain exosomes for 14 days in vitro and could retain exosomes for at least 7 days in vivo. They also found that the 3D printed scaffold could recruit chondrocytes, which was mainly attributed to ECM, and that exosomes could further enhance this effect.

\section{Future perspective}

\section{Drug-loading techniques}

The cell-friendly biological feature of exosome decides that the future drug application via exosome may provide a higher delivery efficiency. The recent research tend is to modify the exosome-secreting cells with transfection to load miRNA(s) into exosomes, thus can be used for OA therapy/cartilage repair [23,44-46]. However, the transfection method in loading RNA has many limitations, such as the unstable productivity of RNA and the unclear factors influencing the RNA level and loading [75], which means the application is still far from clinic. Other drug loading techniques applied for $\mathrm{OA}$ treatment/cartilage repair have not been reported yet, therefore it is significant to develop new drug loading technologies. One of the direction is to use nanoparticles, which could improve the drug loading capacity of exosomes [62]. All in all, to explore the cargo-loading mechanism of exosomes and search for more efficient and stable drug loading technology, will accelerate the development of exosome-based OA therapy/cartilage repair.

\section{Local sustained release system}

Most recent studies demonstrate that exosomes play a significant role in OA therapy/cartilage repair both in animal models and preclinical trials. Nevertheless, the current therapy requests repeatedly local administration for maintaining the effective concentration, which increase pain and the risk of side effects to the subjects. To solve this problem, local controlled release of exosomes with bio-scaffold has begun to be valued [74]. Controlled delivery of therapeutic agents on local joint lesion possesses two advantages. One is that controlling the degradation time of drug vehicle/bio-degradable scaffold and maintaining the effective concentration of drugs, thus prolonging the functional time of drugs at desired dosages. The other is that it will be helpful to combine the exosome-working-platform with other sustained drug release technologies according to the degrees of cartilage injury and osteoarthritis, which can be designed and become personalized therapeutics in the future.

\section{Personalized and "point-to-point" treatment}

Over the past few years, the arrival of liquid biopsy technology has made the generating a database of OA patients much easier, including information concerning exosomes. With the gradual understanding of the mechanism of exosome involved in $\mathrm{OA}$ and adequate information from the database, the patient's articular condition can be analyzed individually. It would be possible to assemble the needed cargos and drugs into the modularized exosomes to achieve personalized treatment with maximizing the therapeutic effect. In addition, the key concept of precision medicine is to treat or "ablate" the pathological condition without damage to normal tissues. As a consequence, exosomes will be a poetical biomarker and therapeutic tool for personalized and precision treatment of OA therapy/cartilage repair in the future.

\section{Conclusion}

Exosomes derived from chondrocytes, synovial cells, and synovial fluid have been shown to be involved in the pathogenesis of OA. Meanwhile many studies have shown that exosomes from natural cells, especially MSCs, could maintain chondrocyte homeostasis and ameliorate the pathological severity of OA, demonstrating the potential therapeutic effect of exosomes for OA/cartilage injury. In addition, exosomes from modified cells with drug loading technologies have been shown improved therapeutic effect. Tissue engineering techniques are also used in the exosome-based OA therapy/cartilage repair. Biological scaffolds, especially hydrogel, have been 
shown to have a good sustained exosome-release effect in cartilage repair. The 3D printing technology can be used to construct more reasonable 3D culture microenvironments for exosomes, and can contribute to design scaffolds with more optimized geometric structure. We believe that drug loading, sustained release and individualized treatment will be the main directions of exosome-based OA therapy/cartilage repair in the near future.

\section{Acknowledgements}

This work was supported by the National Key R\&D Program of China (2019YFA0111900 to YJ), Key Scientific and Technological Innovation Group of Zhejiang Province (2013TD11 to HOY), Zhejiang Province grants (LY17H060003 to JP), NSFC grants (81201395 to YC), and the Dr. Li Dak Sum \& Yip Yio Chin Development Fund for Regenerative Medicine.

\section{Competing Interests}

The authors have declared that no competing interest exists.

\section{References}

1. Sinusas K.Osteoarthritis: diagnosis and treatment. Am Fam Physician. 2012;85(1):49-56

2. Park S, Choi Y, Jung N, Yu Y, Ryu KH, Kim HS, et al. Myogenic differentiation potential of human tonsil-derived mesenchymal stem cells and their potential for use to promote skeletal muscle regeneration. Int J Mol Med. 2016; 37(5):1209-20.

3. Jo CH, Lee YG, Shin WH, Kim H, Chai JW, Jeong EC, et al. Intra-articular injection of mesenchymal stem cells for the treatment of osteoarthritis of the knee: a proof-of-concept clinical trial. Stem Cells. 2014; 32(5): 1254-66.

4. Pashoutan Sarvar D, Shamsasenjan K, Akbarzadehlaleh P. Mesenchymal Stem Cell-Derived Exosomes: New Opportunity in Cell-Free Therapy. Adv Pharm Bull. 2016;6(3):293-299.

5. Mathieu M, Martin-Jaular L, Lavieu G, Théry C. Specificities of secretion and uptake of exosomes and other extracellular vesicles for cell-to-cell communication. Nat Cell Biol. 2019; 21(1): 9-17.

6. Ailawadi S, Wang X, Gu H, Fan GC. Pathologic function and therapeutic potential of exosomes in cardiovascular disease. Biochim Biophys Acta. 2015; 1852(1):1-11

7. Colombo M, Raposo G, Théry C. Biogenesis, secretion, and intercellular interactions of exosomes and other extracellular vesicles. Annu Rev Cell Dev Biol. 2014; 30: 255-89.

8. Kao CY, Papoutsakis ET. Extracellular vesicles: exosomes, microparticles, their parts, and their targets to enable their biomanufacturing and clinical applications. Curr Opin Biotechnol. 2019; 60: 89-98.

9. Zou W, Lai M, Zhang Y, Zheng L, Xing Z, Li T, Zou Z, Song Q, Zhao X, Xia L, Yang J, Liu A, Zhang H2, Cui ZK, Jiang Y, Bai X. Exosome Release Is Regulated by mTORC1. Adv Sci (Weinh). 2018; 6(3): 1801313.

10. Théry C, Ostrowski M, Segura E. Membrane vesicles as conveyors of immune responses. Nat Rev Immunol. 2009;9(8):581-93.

11. Farahat MN, Yanni G, Poston R, Panayi GS. Cytokine expression in synovial membranes of patients with rheumatoid arthritis and osteoarthritis. Ann Rheum Dis. 1993 ; 52(12):870-5.

12. Little CB, Barai A, Burkhardt D, Smith SM, Fosang AJ, Werb Z, Shah M, Thompson EW. Matrix metalloproteinase 13-deficient mice are resistant to osteoarthritic cartilage erosion but not chondrocyte hypertrophy or osteophyte development. Arthritis Rheum. 2009;60(12):3723-33.

13. Kolhe R, Hunter M, Liu S, Jadeja RN, Pundkar C, Mondal AK, Mendhe B, Drewry M, Rojiani MV, Liu Y, Isales CM, Guldberg RE, Hamrick MW, Fulzele S.Gender-specific differential expression of exosomal miRNA in synovial fluid of patients with osteoarthritis. Sci Rep. $2017 ; 7(1): 2029$.

14. Domenis R, Zanutel R, Caponnetto F, Toffoletto B, Cifù A, Pistis C, Di Benedetto P, Causero A, Pozzi M, Bassini F, Fabris M, Niazi KR, Soon-Shiong P, Curcio F. Characterization of the Proinflammatory Profile of Synovial Fluid-Derived Exosomes of Patients with Osteoarthritis. Mediators Inflamm. 2017; 2017: 4814987

15. Song JE, Shin JH, Moon KW, Shon SH, Park JS, Lee E.B, Song YW, Lee EY. A Role of Synovial Exosomes in Osteoclast Differentiation of Inflammatory Arthritis. Annals of the Rheumatic Diseases. 2016; 75 (Suppl 2) :921.
16. Nakasa T, Miyaki S, Kato T, Takada T, Nakamura Y, Ochi M. Exosome derived from osteoarthritis cartilage induces catabolic factor gene expressions in synovium. In: ORS 2016 Annual Meeting, San Francisco; 2012. Trans Orth Res Soc 2012: Abstract 708

17. Kato T, Miyaki S, Ishitobi H, Nakamura Y, Nakasa T, Lotz MK, Ochi M. Exosomes from IL-1 $\beta$ stimulated synovial fibroblasts induce osteoarthritic changes in articular chondrocytes. Arthritis Res Ther. 2014; 16(4):R163.

18. Zhou F, Wang W, Xing Y, Wang T, Xu X, Wang J. NF-kB target microRNAs and their target genes in TNFa-stimulated HeLa cells. Biochim Biophys Acta. 2014;1839(4):344-54.

19. Zhang Z, Kang Y, Zhang Z, Zhang H, Duan X, Liu J, Liu J, Li X, Liao W. Expression of microRNAs during chondrogenesis of human adipose-derived stem cells. Osteoarthritis Cartilage. 2012; 20(12):1638-46.

20. Ni Z, Kuang L, Chen H, Xie Y, Zhang B, Ouyang J, Wu J, Zhou S, Chen L, Su $\mathrm{N}$, Tan Q, Luo X, Chen B, Chen S, Yin L, Huang H, Du X, Chen L. The exosome-like vesicles from osteoarthritic chondrocyte enhanced mature IL-1 $\beta$ production of macrophages and aggravated synovitis in osteoarthritis. Cell Death Dis. 2019; 10(7): 522

21. Meng F, Li Z, Zhang Z, Yang Z, Kang Y, Zhao X, Long D, Hu S, Gu M, He S, $\mathrm{Wu} \mathrm{P}$, Chang $\mathrm{Z}$, He A, Liao W. MicroRNA-193b-3p regulates chondrogenesis and chondrocyte metabolism by targeting HDAC3. Theranostics. 2018;8(10):2862-2883

22. Mao G, Zhang Z, Hu S, Zhang Z, Chang Z, Huang Z, Liao W, Kang Y. Exosomes derived from miR-92a-3p-overexpressing human mesenchymal stem cells enhance chondrogenesis and suppress cartilage degradation via targeting WNT5A. Stem Cell Res Ther. 2018;9(1):247.

23. Hosseini-Farahabadi S, Geetha-Loganathan P, Fu K, Nimmagadda S, Yang HJ, Richman JM.Dual functions for WNT5A during cartilage development and in disease. Matrix Biol. 2013;32(5):252-64

24. Mao G, Hu S, Zhang Z, Wu P, Zhao X, Lin R, Liao W, Kang Y. Exosomal miR-95-5p regulates chondrogenesis and cartilage degradation via histone deacetylase 2/8. J Cell Mol Med. 2018; 22(11): 5354-5366.

25. Mao G, Zhang Z, Huang Z, Chen W, Huang G, Meng F, Zhang Z, Kang Y. MicroRNA-92a-3p regulates the expression of cartilage-specific genes by directly targeting histone deacetylase 2 in chondrogenesis and degradation. Osteoarthritis Cartilage. 2017; 25(4): 521-532.

26. Marta Varela-Eirín, Adrián Varela-Vázquez, Amanda Guitián-Caamaño, Susana B. Bravo-López, Carlos Paíno, Raquel Largo, Eduardo Fonseca, Mustapha Kandouz, Trond Aasen, Arantxa Tabernero, Alfonso Blanco, José R. Caeiro, María D. Mayán. Speed of Senescence and joint inflammation via connexin43-positive exosomes released by osteoarthritic chondrocytes. Annals of the Rheumatic Diseases. 2019; 78 (Suppl 2): 959.

27. Zhao $\mathrm{Y}, \mathrm{Xu}$ J. Synovial fluid-derived exosomal lncRNA PCGEM1 as biomarker for the different stages of osteoarthritis . Int Orthop. 2018; 42(12): 2865-2872.

28. Kang Y, Song J, Kim D, Ahn C, Park S,Chun CH, Jin EJ. PCGEM1 stimulates proliferation of osteoarthritic synoviocytes by acting as a sponge for miR-770. J Orthop Res. 2016; 34(3): 412-8.

29. Wu P, Zhang B, Shi H, Qian H, Xu W.MSC-exosome: A novel cell-free therapy for cutaneous regeneration. Cytotherapy. 2018;20(3):291-301.

30. Tao SC, Yuan T, Rui BY, Zhu ZZ, Guo SC, Zhang CQ.Exosomes derived from human platelet-rich plasma prevent apoptosis induced by glucocorticoid-associated endoplasmic reticulum stress in rat osteonecrosis of the femoral head via the Akt/Bad/Bcl-2 signal pathway. Theranostics. 2017;7(3):733-750.

31. Zhang ZG, Buller B, Chopp M. Exosomes - beyond stem cells for restorative therapy in stroke and neurological injury.Nat Rev Neurol. 2019;15(4):193-203.

32. Cosenza S, Ruiz M, Toupet K, Jorgensen C, Noël D. Mesenchymal stem cells derived exosomes and microparticles protect cartilage and bone from degradation in osteoarthritis. Sci Rep. 2017; 7(1): 16214

33. Zhu H, Ji. J, Fu.T, Yang.J, Gu.Z. The effect of exosomes from bone marrow mesenchymal stem cells on osteoarthritis. Annals of the Rheumatic Diseases; 2018; 77 (Suppl 2): 893

34. Qi H, Liu DP, Xiao DW, Tian DC, Su YW, Jin SF. Exosomes derived from mesenchymal stem cells inhibit mitochondrial dysfunction-induced apoptosis of chondrocytes via p38, ERK, and Akt pathways.In Vitro Cell Dev Biol Anim. $2019 ; 55(3): 203-210$.

35. Zhang S, Chu WC, Lai RC, Lim SK, Hui JH, Toh WS. Exosomes derived from human embryonic mesenchymal stem cells promote osteochondral regeneration. Osteoarthritis Cartilage. 2016; 24(12):2135-2140.

36. Wang Y, Yu D, Liu Z, Zhou F, Dai J, Wu B, Zhou J, Heng BC, Zou XH, Ouyang $\mathrm{H}$, Liu H. Exosomes from embryonic mesenchymal stem cells alleviate osteoarthritis through balancing synthesis and degradation of cartilage extracellular matrix. Stem Cell Res Ther.2017; 14;8(1):189.

37. Zhang S, Teo KYW, Chuah SJ, Lai RC, Lim SK, Toh WS. MSC exosomes alleviate temporomandibular joint osteoarthritis by attenuating inflammation and restoring matrix homeostasis. Biomaterials. 2019Apr; 200:35-47.

38. Tofiño-Vian M, Guillén MI, Pérez Del Caz MD, Castejón MA, Alcaraz MJ. Extracellular Vesicles from Adipose-Derived Mesenchymal Stem Cells Downregulate Senescence Features in Osteoarthritic Osteoblasts. Oxid Med Cell Longev. 2017; 2017:7197598.

39. Tofiño-Vian M, Guillén MI, Pérez Del Caz MD, Silvestre A, Alcaraz MJ. Microvesicles from Human Adipose Tissue-Derived Mesenchymal Stem Cells as a New Protective Strategy in Osteoarthritic Chondrocytes. Cell Physiol Biochem. 2018;47(1):11-25. 
40. Wu J, Kuang L, Chen C, Yang J, Zeng WN, Li T, Chen H, Huang S, Fu Z, Li J, Liu R, Ni Z, Chen L, Yang L. miR-100-5p-abundant exosomes derived from infrapatellar fat pad MSCs protect articular cartilage and ameliorate gait abnormalities via inhibition of mTOR in osteoarthritis. Biomaterials. 2019; 206:87-100.

41. Liu Y, Zou R, Wang Z, Wen C, Zhang F, Lin F. Exosomal KLF3-AS1 from hMSCs promoted cartilage repair and chondrocyte proliferation in osteoarthritis. Biochem J. 2018;475(22):3629-3638.

42. Zhu Y, Wang Y, Zhao B, Niu X, Hu B, Li Q, Zhang J, Ding J, Chen Y, Wang Y. Comparison of exosomes secreted by induced pluripotent stem cell-derived mesenchymal stemcells and synovial membrane-derived mesenchymal stem cells for the treatment of osteoarthritis. Stem Cell Res Ther. 2017; 8(1):64.

43. Chen Y, Xue K, Zhang X, Zheng Z, Liu K. Exosomes derived from mature chondrocytes facilitate subcutaneous stable ectopic chondrogenesis of cartilage progenitor cells. Stem Cell Res Ther. 2018;9(1):318.

44. Liu Y, Lin L, Zou R, Wen C, Wang Z, Lin F. MSC-derived exosomes promote proliferation and inhibit apoptosis of chondrocytes via IncRNA-KLF3-AS1/miR-206/GIT1 axis in osteoarthritis. Cell Cycle. 2018; 17(21-22):2411-2422.

45. Tao SC, Yuan T, Zhang YL, Yin WJ, Guo SC, Zhang CQ. Exosomes derived from miR-140-5p-overexpressing human synovial mesenchymal stem cells enhance cartilage tissue regeneration and prevent osteoarthritis of the knee in a rat model. Theranostics. 2017;7(1):180-195.

46. Sun $\mathrm{H}, \mathrm{Hu} \mathrm{S}$, Zhang Z, Lun J, Liao W, Zhang Z. Expression of exosomal microRNAs during chondrogenic differentiation of human bone mesenchymal stem cells. J Cell Biochem. 2019; 120(1):171-181.

47. Wang $\mathrm{R}, \mathrm{Xu} B, \mathrm{Xu}$ H.TGF- $\beta 1$ promoted chondrocyte proliferation by regulating Sp1 through MSC-exosomes derived miR-135b.Cell Cycle. 2018;17(24).

48. Bissell MJ, Hines WC. Why don't we get more cancer? A proposed role of the microenvironment in restraining cancer progression. Nat Med. 2011; 17(3):320-9

49. Lucchetti D, Calapà F, Palmieri V, Fanali C, Carbone F, Papa A, De Maria R1, De Spirito M, Sgambato A. Differentiation Affects the Release of Exosomes from Colon Cancer Cells and Their Ability to Modulate the Behavior of Recipient Cells. Am J Pathol. 2017; 187(7):1633-1647.

50. Rocha S, Carvalho J, Oliveira P, Voglstaetter M, Schvartz D, Thomsen AR, Walter N, Khanduri R, Sanchez JC, Keller A, Oliveira C, Nazarenko I. 3D Cellular Architecture Affects MicroRNA and Protein Cargo of Extracellular Vesicles. Adv Sci (Weinh). 2018; 6(4):1800948.

51. Villasante A, Marturano-Kruik A, Ambati SR, Liu Z, Godier-Furnemont A, Parsa H, Lee BW, Moore MA4, Vunjak-Novakovic G. Recapitulating the Size and Cargo of Tumor Exosomes in a Tissue-Engineered Model. Theranostics. 2016; 6(8):1119-30

52. Zhang Y, Chopp M, Zhang ZG, Katakowski M, Xin H, Qu C, Ali M, Mahmood A, Xiong Y. Systemic administration of cell-free exosomes generated by human bone marrow derived mesenchymal stem cells cultured under 2D and 3D conditions improves functional recovery in rats after traumatic brain injury. Neurochem Int. 2017; 111:69-81.

53. Kim M, Yun HW, Park DY, Choi BH, Min BH. Three-Dimensional Spheroid Culture Increases Exosome Secretion from Mesenchymal Stem Cells. Tissue Eng Regen Med. 2018; 15(4):427-436.

54. Miceli V, Pampalone M, Vella S, Carreca AP, Amico G, Conaldi PG. Comparison of Immunosuppressive and Angiogenic Properties of Human Amnion-Derived Mesenchymal Stem Cells between 2D and 3D Culture Systems. Stem Cells Int. 2019;2019:7486279.

55. Haraszti RA, Miller R, Stoppato M, Sere YY, Coles A, Didiot MC, Wollacott R, Sapp E, Dubuke ML, Li X, Shaffer SA, DiFiglia M, Wang Y, Aronin N, Khvorova A. Exosomes Produced from 3D Cultures of MSCs by Tangential Flow Filtration Show Higher Yield and Improved Activity. Mol Ther. 2018; 26(12): 2838-2847.

56. Eguchi $T$, Sogawa $C$, Okusha $Y$, Uchibe $K$, Jinuma $R$, Ono $K$, Nakano $K$ Murakami J, Itoh M, Arai K, Fujiwara T, Namba Y, Murata $Y$, Ohyama K, Shimomura M, Okamura H, Takigawa M, Nakatsura T, Kozaki KI, Okamoto K, Calderwood SK. Organoids with cancer stem cell-like properties secrete exosomes and HSP90 in a 3D nanoenvironment. PLoS One. 2018; 13(2):e0191109.

57. Patel DB, Luthers CR, Lerman MJ, Fisher JP, Jay SM. Enhanced extracellular vesicle production and ethanol-mediated vascularization bioactivity via a 3D-printed scaffold-perfusion bioreactor system. Acta Biomater. 2019; 95:236-244.

58. Kasper JY, Hermanns MI, Kraegeloh A, Roth W, Kirkpatrick CJ, Unger RE. In Vitro Entero-Capillary Barrier Exhibits Altered Inflammatory and Exosomal Communication Pattern after Exposure to Silica Nanoparticles. Int J Mol Sci. 2019;20(13). pii: E3301.

59. Roma-Rodrigues C, Pereira F, Alves de Matos AP, Fernandes M, Baptista PV, Fernandes AR.Smuggling gold nanoparticles across cell types - A new role for exosomes in gene silencing. Nanomedicine. 2017; 13(4):1389-1398.13(4):1389-1398

60. Liang J, Zhang X, He S, Miao Y, Wu N, Li J, Gan Y. Sphk2 RNAi nanoparticles suppress tumor growth via downregulating cancer cell derived exosomal microRNA. J Control Release. 2018; 286:348-357.

61. Khongkow M, Yata T, Boonrungsiman S, Ruktanonchai UR, Graham D, Namdee K.Surface modification of gold nanoparticles with neuron-targeted exosome for enhanced blood-brain barrier penetration.Sci Rep. 2019; 9(1):8278
62. Lin $\mathrm{Y}$, Wu J, Gu W, Huang Y, Tong Z, Huang L, Tan J. Exosome-Liposome Hybrid Nanoparticles Deliver CRISPR/Cas9 System in MSCs. Adv Sci (Weinh). 2018;5(4):1700611.

63. Zhang J, Liu X, Li H, Chen C, Hu B, Niu X, Li Q1, Zhao B, Xie Z, Wang Y. Exosomes/tricalcium phosphate combination scaffolds can enhance bone regeneration by activating the PI3K/Akt signaling pathway. Stem Cell Res Ther. 2016; 7(1):136.

64. Tao SC, Guo SC, Li M, Ke QF, Guo YP, Zhang CQ. Chitosan Wound Dressings Incorporating Exosomes Derived from MicroRNA-126-Overexpressing Synovium Mesenchymal Stem Cells Provide Sustained Release of Exosomes and Heal Full-Thickness Skin Defects in a Diabetic Rat Model. Stem Cells Transl Med. 2017; 6(3): 736-747.

65. Han C, Zhou J, Liang C, Liu B, Pan X, Zhang Y, Wang Y, Yan B, Xie W, Liu F, Yu XY, Li Y. Human umbilical cord mesenchymal stem cell derived exosomes encapsulated in functional peptide hydrogels promote cardiac repair. Biomater Sci. 2019; 7(7): 2920-2933.

66. Han C, Zhou J, Liu B, Liang C, Pan X, Zhang Y, Wang Y1, Shao L, Zhu B, Wang J, Yin Q, Yu XY, Li Y. Delivery of miR-675 by stem cell-derived exosomes encapsulated in silk fibroin hydrogel prevents aging-induced vascular dysfunction in mouse hindlimb. Mater Sci Eng C Mater Biol Appl. 2019:99:322-332.

67. Wang $\mathrm{C}$, Wang $\mathrm{M}, \mathrm{Xu} \mathrm{T}$, Zhang $\mathrm{X}$, Lin $\mathrm{C}$, Gao $\mathrm{W}, \mathrm{Xu} H$, Lei B, Mao C. Engineering Bioactive Self-Healing Antibacterial Exosomes Hydrogel for Promoting Chronic Diabetic Wound Healing and Complete Skin Regeneration. Theranostics. 2019; 9(1):65-76.

68. Zhang K, Zhao X, Chen X, Wei Y, Du W, Wang Y, Liu L, Zhao W, Han Z, Kong D, Zhao Q, Guo Z, Han Z, Liu N, Ma F, Li Z. Enhanced Therapeutic Effects of Mesenchymal Stem Cell-Derived Exosomes with an Injectable Hydrogel for Hindlimb Ischemia Treatment. ACS Appl Mater Interfaces. 2018; 10(36):30081-30091.

69. Vega SL, Kwon MY, Burdick JA. Recent advances in hydrogels for cartilage tissue engineering.Eur Cell Mater. 2017;33:59-75

70. Schneider MC, Barnes CA, Bryant SJ. Characterization of the chondrocyte secretome in photoclickable poly(ethylene glycol) hydrogels. Biotechnol Bioeng. 2017;114(9):2096-2108

71. Liu X, Yang Y, Li Y, Niu X, Zhao B, Wang Y, Bao C, Xie Z, Lin Q, Zhu L. Integration of stem cell-derived exosomes with in situ hydrogel glue as a promising tissue patch for articular cartilage regeneration. Nanoscale. 2017, 9(13): 4430-4438.

72. Diomede F, Gugliandolo A, Cardelli P, Merciaro I, Ettorre V, Traini T, Bedini R, Scionti D, Bramanti A, Nanci A, Caputi S, Fontana A, Mazzon E, Trubiani O. Three-dimensional printed PLA scaffold and human gingival stem cell-derived extracellular vesicles: a new tool for bone defect repair. Stem Cell Res Ther. 2018;13;9(1):104.

73. Pizzicannella J, Diomede F, Gugliandolo A, Chiricosta L Bramanti P, Merciaro I, Orsini T, Mazzon E, Trubiani O. 3D Printing PLA/Gingival Stem Cells/ EVs Upregulate miR-2861 and -210 during Osteoangiogenesis Commitment. Int J Mol Sci. 2019;20(13). pii: E3256.

74. Chen P, Zheng L, Wang Y, Tao M, Xie Z, Xia C, Gu C Chen J, Qiu P, Mei S, Ning L, Shi Y, Fang C, Fan S, Lin X. Desktop-stereolithography 3D printing of a radially oriented extracellular matrix/mesenchymal stem cell exosome bioink for osteochondral defect regeneration. Theranostics. 2019; 9(9):2439-2459.

75. Batagov AO, Kuznetsov VA, Kurochkin IV. Identification of nucleotide patterns enriched in secreted RNAs as putative cis-acting elements targeting them to exosome nano-vesicles. BMC Genomics. 2011;12 Suppl 3:S18. 